\section{Alterações Metabólicas da Sindrome Lipodistrófica do HIV}

\section{RESUMO}

A introdução da highly active antiretroviral therapy (HAART) - terapia anti-retroviral fortemente ativa - vem reduzindo a morbidade e a mortalidade em pacientes infectados com o vírus da imunodeficiência humana (HIV). Entretanto, tratamentos prolongados, com combinações de drogas, são de difícil manutenção devido à má aderência e aos efeitos tóxicos. O tratamento com agentes anti-retrovirais, especialmente os inibidores da protease, fez surgir uma síndrome caracterizada por redistribuição anormal da gordura corporal, alterações no metabolismo glicêmico, resistência insulínica e dislipidemia, chamada de síndrome lipodistrófica do HIV (SLHIV). Atualmente não existe nenhum consenso para prevenção ou tratamento da síndrome, cuja causa permanece desconhecida. Esta revisão enfatiza os achados clínicos e dados da literatura a respeito da SLHIV, pois um melhor entendimento desta síndrome por infectologistas, cardiologistas e endocrinologistas é essencial para o manejo da doença. (Arq Bras Endocrinol Metab 2005;49/6:871-881)

Descritores: Síndrome lipodistrófica do HIV; AIDS; HIV; Lipodistrofia; Síndrome metabólica do HIV

\section{ABSTRACT}

HIV Lipodystrophy Syndrome.

The introduction of highly active antiretroviral therapy (HAART) has reduced morbidity and mortality in patients infected with the human immunodeficiency virus (HIV). However, prolonged treatment with combination regimens can be difficult to sustain because of problems with adherence and toxic effects. Treatment with antiretroviral agents - protease inhibitors in particular - has uncovered a syndrome of abnormal fat redistribution, impaired glucose metabolism, insulin resistance and dyslipidemia, collectively termed lipodystrophy syndrome (SLHIV). Nowadays, no clinical guidelines are available for the prevention or treatment of SLHIV, and its cause have yet to be totally elucidated. This review emphasizes the clinical features and the data from previous studies about the SLHIV taking into account that a better understanding of this syndrome for HIV specialists, cardiologists and endocrinologists is fundamental for the disease control. (Arq Bras Endocrinol Metab 2005;49/6:871-881)

Keywords: Lipodystrophy syndrome HIV; AIDS; HIV; Lipodystrophy; Metabolic syndrome HIV

A SÍNDROME DA IMUNODEFICIÊNCIA HUMANA adquirida (SIDA) foi descrita em 1981 nos Estados Unidos (1). Desde o surgimento dos primeiros casos, observou-se uma importante alteração nas características demográficas e epidemiológicas da doença. Inicialmente restrita a áreas específicas, logo atingiu várias regiões do mundo tornando-se uma pandemia.

\section{revisão}

\author{
Angélica M.M. Valente \\ André F. Reis \\ Daisy M. Machado \\ Regina C.M. Succi \\ Antonio R. Chacra
}

\begin{abstract}
Disciplina de Endocrinologia e Metabologia ( $A M M V, A F R$, ARC), Departamento de Medicina, e Disciplina de Infectologia Pediátrica (DMM, RCMS), Departamento de Pediatria, Universidade Federal de São Paulo - Escola Paulista de Medicina, São Paulo, SP.
\end{abstract}

Recebido em 04/02/05

Revisado em 08/07/05 Aceito em 15/09/05 
Segundo dados da Organização Mundial de Saúde (OMS), estima-se que no final de 2002 cerca de 42 milhões de pessoas estariam infectadas pelo vírus da imunodeficiência humana (HIV) em todo mundo (2), dos quais 1,5 milhão somente na América Latina (2). No Brasil, de acordo com os dados do Ministério da Saúde, 257.780 casos de SIDA haviam sido notificados até o final de 2002 (3).

No início da epidemia as relações homossexuais e o uso de drogas endovenosas eram as principais vias de transmissão da doença, representando os chamados "grupos de risco" $(1,2)$. Atualmente, observa-se um aumento da transmissão por relações heterossexuais, sendo esta a principal via de contágio entre as mulheres (2). A modificação do perfil dos indivíduos infectados resultou em um aumento crescente no número de mulheres e, conseqüentemente, de crianças infectadas pelo HIV através da via vertical $(2,3)$.

Com o advento da highly active antiretroviral therapy (HAART) (figuras l e 2), conhecida como terapia anti-retroviral fortemente ativa, observou-se um profundo impacto na história natural da infecção pelo HIV. Desta forma, o emprego de combinações terapêuticas contendo drogas da classe dos Inibidores da Protease (IP) promoveu uma importante e sustentada supressão na replicação viral (4), elevando a sobrevida e a qualidade de vida dos pacientes soropositivos (5).

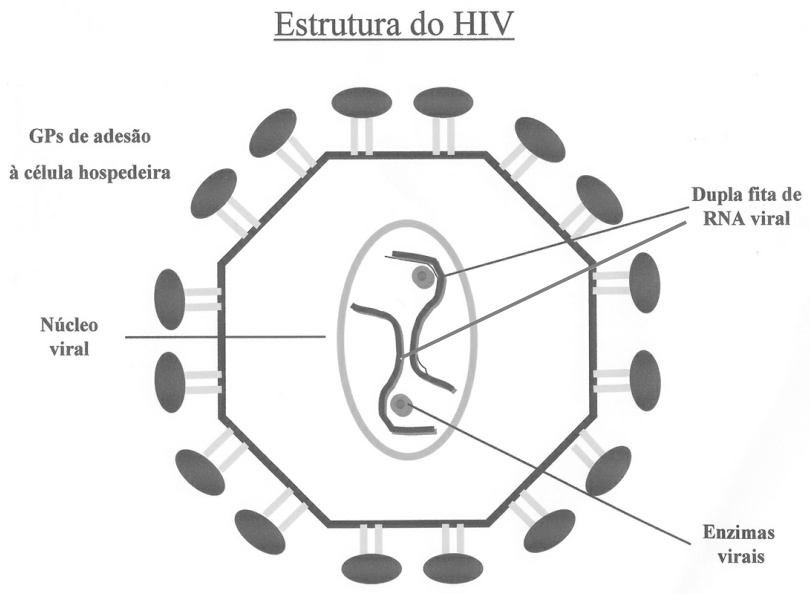

Figura 1. Vírus da Imunodeficiência Humana: Estrutura. $\mathrm{O}$ vírus da imunodeficiência humana (HIV) pertence à família Retroviridae, gênero Lentivirus. Estruturalmente, o HIV constitui-se de partícula icosaédrica, composta de um envelope fosfolipídico, onde estão inseridas proteínas virais e da células hospedeira, incluindo as duas principais glicoproteínas, a gp120 e gp41. Apresenta enzimas que serão fundamentais em seu processo de replicação, em especial a transcriptase reversa e a protease. É através da inibição dessas enzimas virais que agem algumas drogas anti-retrovirais.
No entanto, observou-se que a terapia anti-retroviral é acompanhada de alterações metabólicas como dislipidemia, resistência insulínica, hiperglicemia e redistribuição da gordura corporal (6-10), fatores de risco para doença cardiovascular (8-11). O conjunto destas alterações é conhecido como síndrome lipodistrófica do HIV (SLHIV).

No presente artigo, realizamos uma revisão da literatura sobre a SLHIV com ênfase nas suas alterações metabólicas, nas estratégias para o seu seguimento e no risco cardiovascular de população soropositiva.

\section{SÍNDROME LIPODISTRÓFICA DO HIV (SLHIV)}

\section{Histórico}

A SLHIV foi oficialmente descrita pelo Food and Drug Administration (FDA), órgão norte-americano regulador da liberação e uso de medicamentos, em 1997 (12). Inicialmente, a SLHIV foi denominada de "Crixbelly" (13), pois os primeiros casos de redistribuição da gordura corporal foram observados após a

\section{Mecanismo de Ação dos Antiretrovirais}

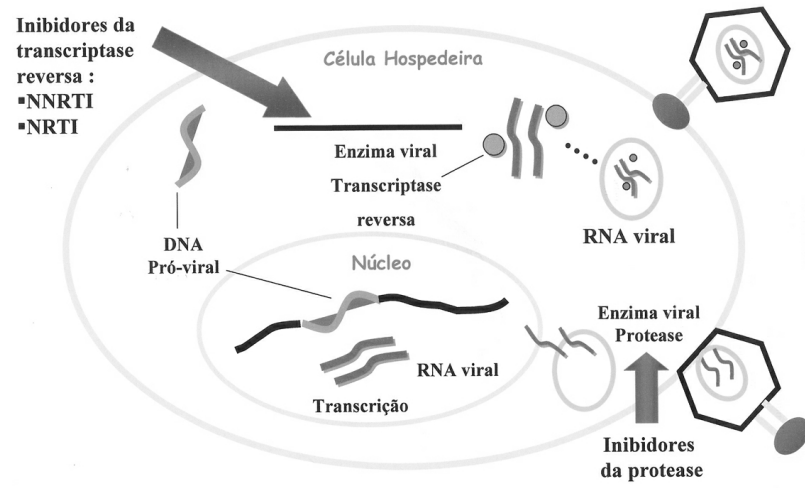

Figura 2. Ciclo de replicação do HIV e mecanismo de ação dos anti-retrovirais.

O HIV, através de suas glicoproteínas de superfície, adere à célula hospedeira e introduz seu material genético no citoplasma da célula. A enzima viral transcriptase reversa transforma a dupla fita de RNA viral em DNA pró-viral, que migra até o núcleo da célula hospedeira unindo-se ao seu material genético. Ocorre o processo de transcrição, pelo qual se formam novas moléculas de RNA viral, as quais migram até o citoplasma e, por ação da enzima viral protease, se unem aos demais componentes virais, havendo a formação de um novo HIV. Desta forma, as drogas da classe dos ínibidores da transcriptase (análogos de nucleosídeos NRTI - ou não análogos de nucleosídeos - NNRTI) inibem a primeira fase da replicação viral através da inibição da enzima viral transcriptase reversa. As drogas da classe dos inibidores da protease impedem a ocorrência da última fase da replicação viral por inibirem a enzima viral protease. 
utilização do Crixivan ${ }^{\circledR}$ (Indinavir), medicamento da classe dos inibidores da protease (IP) (13). A associação entre o uso de Indinavir e redistribuição da gordura corporal foi descrita em 1998, com o emprego de tomografia computadorizada demonstrando o aumento da gordura visceral nestes indivíduos (14). Com o surgimento de novos IPs, concluiu-se que a redistribuição da gordura corporal não era um efeito exclusivo do Indinavir, sendo esta denominação abandonada $(7,9,10)$.

Miller e cols. (15), após observarem as semelhanças clínicas entre pacientes com SLHIV e síndrome de Cushing, passaram a denominá-la de "pseudo-síndrome de Cushing". Porém, estudos posteriores não demonstraram alterações no eixo hipotálamohipófise-adrenal (EHHA) dos pacientes soropositivos para o HIV (16).

Atualmente, algumas sinonímias são empregadas para a SLHIV (17), tais como síndrome da redistribuição da gordura corporal $(18,19)$, síndrome metabólica associada à terapia anti-retroviral (ARV) (10) ou, mais recentemente, lipodistrofia dislipidêmica associada ao HIV/HAART (HADL) (20).

\section{Componentes da SLHIV (tabela 1)}

\section{Alterações Lipídicas}

Antes da introdução da HAART, já existiam relatos de hipertrigliceridemia entre pacientes soropositivos (21); entretanto, após sua utilização, novas alterações no metabolismo lipídico passaram a ser observadas $(7,22,23)$. Ressalta-se que a dislipidemia associada à infecção pelo HIV caracteriza-se por baixos níveis séricos de HDL colesterol e elevação de colesterol total, LDL colesterol e triglicérides, constituindo perfil lipídico sabidamente aterogênico (10).

No entanto, muitos estudos postulam a associação entre hipercolesterolemia e/ou hipertrigliceridemia e uso de IP. Após 5 anos de seguimento de pacientes soropositivos em terapia com IP observou-se uma incidência cumulativa de aproximadamente $20 \%$ de novos casos de hipercolesterolemia e hipertrigliceridemia (24).

Em um estudo canadense, pacientes infectados pelo HIV, virgens de tratamento, foram seguidos por um ano a partir do início da terapia ARV (25); observou-se uma incidência cumulativa de dislipidemia de 9\%, a qual esteve associada somente com a presença de IP na terapia inicial $(25)$.

Entretanto, observou-se que um grupo de pacientes soropositivos que nunca havia recebido IP e que estava em tratamento com inibidores da transcriptase reversa análogos de nucleosídeos (NRTI) apresentava níveis significantemente elevados de triglicérides (26), sugerindo a existência de outros fatores implicados nestas alterações. As duas principais combinações de drogas observadas foram zidovudina-lamivudina e estavudina-lamivudina.

Tabela 1. Componentes da Síndrome Lipodistrófica do HIV (SLHIV).

Dislipidemia

$\Uparrow$ Colesterol total
$\Uparrow \mathrm{LDL}$ colesterol
$\Downarrow \mathrm{HDL}$ colesterol
$\Uparrow$ Triglicérides

Alteraçōes glicêmicas

- Glicemia de jejum alterada (pré-diabetes): Glicemia de jejum 100-125mg/dl / ADA* - Intolerância a glicose: Glicemia 2 horas após sobrecarga oral com glicose $140-199 \mathrm{mg} / \mathrm{dl} / \mathrm{ADA}$ *

- Diabetes mellitus: Glicemia de jejum $\geq 126 \mathrm{mg} / \mathrm{dl}$ ou glicemia 2 horas após sobrecarga oral com glicose $\geq 200 \mathrm{mg} / \mathrm{dl} / \mathrm{ADA}^{*}$

- Resistência insulínica

Lipodistrofia

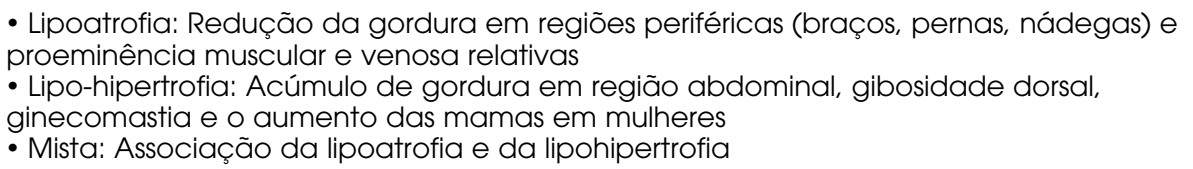

Doença cardiovascular

Resistência insulínica

* ADA: American Diabetes Association. 
Indivíduos saudáveis não infectados pelo HIV que receberam ritonavir (IP) durante 2 semanas, apresentaram elevação nos níveis séricos de colesterol total, triglicérides, lipoproteína (A) e lipoproteína B (27), sugerindo que tais anormalidades poderiam ocorrer independentemente da infecção pelo vírus.

Um recente estudo demonstrou a presença de hipercolesterolemia em $27 \%$ dos indivíduos em terapia anti-retroviral com IP, em $23 \%$ dos pacientes que recebiam somente inibidores da transcriptase reversa não análogos de nucleosídeos (NNRTI) e 10\% no grupo tratado apenas com NRTI, quando comparado com $8 \%$ nos indivíduos sem terapia anti-retroviral (28). A correspondência destes dados para hipertrigliceridemia foi de $40 \%, 32 \%$ e $23 \%$, respectivamente, quando comparado com os $15 \%$ dos indivíduos que não recebiam tratamento (28).

$\mathrm{Na}$ ausência de um consenso específico para o tratamento da dislipidemia nos indivíduos soropositivos, utilizam-se os mesmos critérios do National Cholesterol Education Program (NCEP) para a população geral $(29,30)$. Todos os pacientes adultos infectados pelo HIV necessitam de dosagem anual de lípides antes do início da terapia anti-retroviral e a cada 2 meses após a instituição do tratamento ou após qualquer mudança em seu esquema terapêutico (31). É importante a investigação da presença de história familiar para dislipidemia e/ou diabetes, bem como dos hábitos de vida do paciente: uso de álcool ou medicamentos como estrógeno. Desta forma, a mudança no estilo de vida torna-se indispensável no tratamento da SLHIV (31). As estatinas como a sinvastatina, lovastatina e atorvastatina são metabolizadas pelo citocromo P-450 isoforma $3^{\mathrm{A}} 4$, o qual é inibido pelos IPs (32); entretanto, a pravastatina e o ezetimibe podem ser boas alternativas pelo metabolismo alternativo (32). Nos casos de associação entre drogas antiretrovirais e antilipemiantes (fibratos e/ou estatinas) é fundamental uma rigorosa monitorização da função renal, das enzimas hepáticas e da creatinofosfoquinase (CPK) pela potencialização dos efeitos nefrotóxicos, hepatotóxicos e miotóxicos destas medicações (10).

\section{Alterações glicêmicas e resistência insulínica}

Em maio de 1997, o FDA relatou a ocorrência de 83 casos de hiperglicemia em pacientes soropositivos em uso de IP (12), sugerindo uma possível associação entre esta classe de medicamentos e distúrbios glicêmicos.

A prevalência de novos casos de diabetes varia de l a $6 \%$ entre os pacientes soropositivos tratados com IP (10), aproximando-se da prevalência estimada em várias populações. Entretanto, entre os usuários de IP, obser- va-se uma maior ocorrência de resistência insulínica sem desenvolvimento de diabetes mellitus (33).

$\mathrm{O}$ tratamento de indivíduos não infectados pelo HIV com indinavir - inibidor da protease - promoveu instalação rápida de resistência insulínica, sem mudanças na composição corporal (34). Contudo, é importante salientar que a resistência insulínica pode estar associada à própria infecção pelo HIV, provavelmente por ação direta do vírus na função das células $\beta$ pancreáticas, assim como nos mecanismos de secreção de insulina (35).

Recomenda-se que a glicemia de jejum seja realizada anualmente em pacientes soropositivos ainda sem terapia anti-retroviral, e poucas semanas após a introdução da terapia ou após qualquer mudança no esquema das drogas. $\mathrm{O}$ teste oral de tolerância à glicose ou a dosagem da insulinemia de jejum deverão ser realizados em pacientes soropositivos que apresentarem outro fator de risco cardiovascular ou naqueles com história familiar de diabetes tipo 2 (31). Quanto ao tratamento, há sugestões de que o uso de metformina em pacientes soropositivos em terapia antiretroviral promova redução na glicemia de jejum, na gordura visceral e na resistência insulínica (36-38). Da mesma forma, o uso de metformina em indivíduos infectados pelo HIV reduz os níveis de marcadores séricos de disfunção endotelial (PAI-l e tPA), os quais podem encontrar-se elevados neste grupo de pacientes $(36,37)$. É importante salientar que o uso concomitante de metformina e anti-retrovirais da classe NRTIs pode elevar o risco de ocorrência de acidose lática (evento adverso dos NRTIs ), devendo ser utilizados com cautela e rigorosa monitorização (39).

Em 2004, realizou-se um estudo randomizado, duplo-cego, com 3 meses de seguimento, que avaliou os efeitos da rosiglitazona $(4 \mathrm{mg} / \mathrm{dia})$ na hiperinsulinemia e na lipoatrofia de 28 pacientes soropositivos em terapia anti-retroviral. Encontrou-se melhora significante na sensibilidade insulínica, elevação nos níveis de adiponectina, redução dos ácidos graxos livres e aumento da gordura periférica com o uso da medicação em relação ao grupo placebo (40). Entretanto, outro estudo australiano, de desenho semelhante, utilizou a mesma droga durante 12 meses e não observou alterações significativas na composição corporal (41). Assim, novos estudos são necessários para confirmar o benefício e a segurança da utilização desta classe de medicamentos em pacientes infectados pelo HIV.

\section{Lipodistrofia do HIV}

A lipodistrofia do HIV caracteriza-se pela redistribuição da gordura corporal em pacientes soroposi- 
tivos. Apesar de fortemente associada ao uso dos IPs, alguns NRTIs, especialmente a estavudina $\left(\mathrm{d}_{4} \mathrm{~T}^{\circledR}\right)$, também podem estar implicados em sua gênese $(42,43)$.

A lipodistrofia pode ser classificada clinicamente em 3 categorias: 1 - lipoatrofia: caracterizada pela redução da gordura em regiões periféricas como braços, pernas, face e nádegas, podendo apresentar proeminência muscular e venosa relativas; 2 - lipohipertrofia: caracterizada pelo acúmulo de gordura em região abdominal, presença de gibosidade dorsal, ginecomastia e aumento das mamas em mulheres; 3 forma mista: caracterizada pela associação de componentes das duas formas anteriormente descritas (44).

A prevalência de lipodistrofia em usuários de IP é cerca de $64 \%$ e de $10,5 \%$ em homens e mulheres, respectivamente $(18,23)$.

Tsiodras e cols. (24) avaliaram 221 indivíduos soropositivos em terapia com IP e encontraram uma incidência cumulativa de novos casos de lipodistrofia da ordem de 13\%, estando os IPs independentemente associados com todos os componentes da síndrome.

Outra análise prospectiva acompanhando 366 pacientes soropositivos durante um ano, a partir do início da HAART, demonstrou uma incidência cumulativa de $29 \%$ para lipoatrofia, 23\% lipo-hipertrofia e $13 \%$ da lipodistrofia forma mista (25). Este estudo reforçou a hipótese de que alterações morfológicas e lipídicas são comuns entre indivíduos que iniciaram pela primeira vez o uso da terapia anti-retroviral. Nesta análise, o uso dos IPs associou-se com todas as formas de lipodistrofia.

A despeito de muitos estudos demonstrarem a relação entre IP e lipodistrofia, alguns autores observaram que pacientes soropositivos sem terapia anti-retroviral ou mesmo com esquemas sem IP também apresentavam tais alterações, levantando a hipótese da existência de outras etiologias para a lipodistrofia do HIV.

Um estudo recente comparou a síndrome lipodistrófica (SL) de usuários de IP (SL-IP) com a SL de pacientes utilizando NRTI e sem IP (SL-NRTI) (42). Observou-se a ocorrência da SL em ambos os grupos, sendo que a SL-NRTI diferiu da SL-IP pelo início precoce de sintomas e perda de peso; maiores níveis de lactato e alanina aminotransferase; e menor albumina, colesterol, triglicérides, glicose e insulina (42).

Os pacientes em uso de HAART apresentam, em sua maioria, um bom controle da doença avaliado através de marcadores biológicos representados pelo número de linfócitos CD4 e contagem da carga viral $(4,5)$. Sendo assim, a suspensão, ou até mesmo a substituição do esquema terapêutico, representa uma decisão difícil. Entretanto, a ocorrência de alterações metabólicas que elevam o risco cardiovascular, bem como de alterações estéticas (secundárias à lipodistrofia e responsáveis por importantes problemas psicológicos), determinam o dilema de modificar ou não o esquema anti-retroviral. No intuito de analisar os efeitos da substituição de IP, avaliou-se 81 pacientes em terapia com IP, com lipodistrofia predominantemente do tipo lipoatrofia periférica. Um grupo teve seus IPs substituídos por outras classes de drogas antiretrovirais, enquanto o outro grupo manteve a terapia vigente. Após 24 semanas de acompanhamento concluiu-se que, predominantemente nos pacientes com lipoatrofia, a troca do IP reduziu os níveis lipídicos e a gordura abdominal; entretanto, diminuiu também a gordura periférica e não alterou de forma significativa a resistência insulínica. O controle virológico destes pacientes manteve-se inalterado após a substituição terapêutica (45). Entretanto, devido à magnitude da decisão, novos estudos se fazem necessários.

A fisiopatologia da SLHIV ainda não foi totalmente elucidada. Assim sendo, torna-se difícil o estabelecimento da terapêutica mais eficaz. Estuda-se o efeito de dietas e programas de atividade física na lipodistrofia $(46,47)$. O uso de metformina, na presença de outras alterações metabólicas, parece ser uma opção terapêutica promissora (36-38). Existem alguns relatos de caso utilizando hormônio do crescimento (GH) nesta população; contudo, há controvérsias quanto aos efeitos do GH na homeostase glicídica e no desenvolvimento de neoplasias malignas, às quais estes pacientes já são mais susceptíveis (48). Uma alternativa para os casos de lipodistrofia seria o emprego de procedimentos estéticos como a cirurgia plástica, opção paliativa e que não interfere no perfil metabólico da síndrome (49). Uma droga da classe dos IPs recentemente descoberta, chamada atazanavir, caracteriza-se por sua potência antiviral e segurança, não alterando níveis séricos de colesterol total e triglicérides em pacientes virgens de tratamento antiretroviral $(49,50)$. Estudos demonstram, ainda, que o atazanavir reduziu os níveis de colesterol total, LDL colesterol, triglicérides, gordura abdominal e gibosidade dorsal de indivíduos já em tratamento com a HAART (50-53), surgindo como alternativa para os casos de SLHIV.

\section{Alterações Cardiovasculares na SIDA}

O coração é um órgão bastante afetado nos pacientes soropositivos (54). Entretanto, desde o surgimento da HAART, as características das alterações cardiovasculares modificaram-se substancialmente (54). 
Antes do advento da HAART, as manifestações cardiovasculares mais comuns incluíam cardiomiopatia dilatada, endocardites, miocardites, pericardites, ICC direita causada por hipertensão pulmonar, alterações no sistema de condução do coração e neoplasias infiltrativas como linfomas ou sarcoma de Kaposi (55-57). Desde a introdução da HAART em 1996, observou-se um declínio das patologias acima citadas e o surgimento de artigos relatando a ocorrência de doença arterial coronariana em pacientes soropositivos jovens em uso de IP $(58,59)$, apontando para a possibilidade de que estes pacientes apresentariam risco aumentado para doença cardiovascular (60-62).

Estudos necroscópicos demonstraram a associação entre doença arterial coronariana e $\operatorname{HIV}(63,64)$. Relatos de necrópsias mesmo em crianças infectadas pelo HIV, com idade variando de 13 meses a 7 anos, já demonstraram a presença de doença arterial coronariana (63). A análise microscópica destas artérias coronárias revela a presença de reação inflamatória no endotélio, com linfócitos e células mononucleares gigantes, com fragmentação das fibras de elastina e fibrose intimal resultando em redução da luz do vaso, sendo justamente estas alterações que predispõem a ocorrência de eventos coronarianos agudos (65-66). Um outro estudo necroscópico em pacientes soropositivos com idade média de 27 anos evidenciou a presença de lesões ateroscleróticas (64).

O Frankfurt Cohort Study, uma análise retrospectiva de 4.993 pacientes soropositivos em uso de HAART, mostrou que a incidência de infarto agudo do miocárdio por 1.000 pacientes/ano aumentou de 0,86 no período de 1983 a 1986 para 3,41 no período de 1995 a 1998, coincidindo com a introdução da HAART (59).

Analisando-se a taxa de hospitalização por doença arterial coronariana e IAM de indivíduos infectados pelo HIV, em uso de IP, não se observa um aumento na incidência de eventos cardiovasculares (67).

Bozzette e cols. (68) realizaram um estudo prospectivo com o objetivo de avaliar o risco de doença cardiovascular e cerebrovascular entre 36.766 pacientes soropositivos, e também avaliar a relação entre o risco destas doenças e a HAART. O tempo de acompanhamento foi de aproximadamente 8 anos, avaliando os pacientes antes e depois da introdução da HAART. Após o início da terapia ARV, a taxa de admissão por doença cardiovascular e cerebrovascular diminuiu de 1,7/100 pacientes/ano em 1995 (era pré-HAART) para $0,9 / 100$ pacientes/ano em 2001, não sustentando a hipótese de aumento de eventos ou morte por doença coronariana e cerebrovascular após a introdução da terapia ARV.
Calculou-se o risco acumulado de doença cardiovascular em 10 anos de observação em pacientes soropositivos com alteração da distribuição da gordura corporal comparando-se com participantes do Framingham Offspring Study (FOS) (19). A doença $\mathrm{CV}$ neste período esteve significativamente elevada entre os pacientes soropositivos com redistribuição da gordura corporal, quando comparados com a população do FOS. Neste estudo, o aumento do risco relativo de doença $\mathrm{CV}$ em 10 anos foi maior entre os pacientes com lipoatrofia; no entanto, o uso de IP não conferiu um aumento adicional no risco estimado de doença CV. Indivíduos soropositivos sem redistribuição de gordura não demonstraram elevação no risco de 10 anos quando comparado com controles pareados do FOS.

No final de 2003, foi publicado um importante estudo para avaliar o risco cardiovascular em indivíduos infectados pelo HIV. Trata-se do The Data Collection on Adverse Events of Anti-HIV Drugs (DAD) Study Group (69), um estudo prospectivo que acompanhou 23.468 pacientes, dos quais $126(0,5 \%)$ apresentaram infarto agudo do miocárdio (IAM). A incidência de IAM aumentou com o tempo de exposição à terapia anti-retroviral. Outros fatores significativamente associados ao IAM foram idade, tabagismo, doença cardiovascular prévia, sexo masculino, hipercolesterolemia, hipertrigliceridemia e diabetes. Não estiveram associados com IAM: presença de antecedente familiar para doença cardiovascular, lipodistrofia e elevação da pressão arterial. Os autores concluíram que a terapia anti-retroviral esteve independentemente associada com, aproximadamente, $26 \%$ de aumento relativo na taxa de IAM, por ano de exposição, durante os primeiros 4 a 6 anos de uso da terapia anti-retroviral. Entretanto, o risco absoluto de IAM foi menor do que os comprovados benefícios do tratamento anti-retroviral (69).

\section{Adiponectina e Leptina na Síndrome Lipodistrófica do HIV}

Os adipócitos são células hormonal e metabolicamente ativas, secretando proteínas como o PAI- $1, \mathrm{TNF} \alpha, \mathrm{IL}_{6}$, leptina e adiponectina (70-74). Dentre elas, os níveis de adiponectina correlacionam-se inversamente com a obesidade, resistência insulínica, diabetes tipo 2, doença cardiovascular e lipodistrofia congênita e adquirida em indivíduos não infectados pelo HIV (75-77).

Em um estudo com 112 pacientes soropositivos tratados com HAART (78), observou-se níveis maiores de adiponectina em pacientes sem lipodistrofia em comparação com os indivíduos que apresentavam 
redistribuição da gordura corporal. Este estudo sugere que a adiponectina estaria inversamente correlacionada com massa de gordura visceral, triglicérides, resistência insulínica e diretamente associada com HDL colesterol e gordura periférica em pacientes soropositivos tratados com HAART.

A leptina é um hormônio produto do gene obese (ob), sintetizada e secretada pelos adipócitos (79). Já é bem estabelecido que em indivíduos não infectados pelo HIV a leptinemia de jejum correlaciona-se positivamente com o acúmulo de gordura central e total e com a resistência insulínica $(71,81)$. Recentemente tem-se observado que a reposição de leptina em adultos com síndromes lipodistróficas adquiridas e congênitas (não relacionadas com HIV) resulta em melhora do controle glicêmico, redução dos níveis de triglicérides e, até mesmo, suspensão da terapia antidiabética oral (82).

Neste sentido, avaliou-se a relação da leptina e da resistência insulínica com a redistribuição da gordura corporal em pacientes soropositivos, em terapia antiretroviral por um período de 6 meses $(80)$. Baixos níveis de leptina foram independentemente associados com resistência insulínica em pacientes com lipodistrofia do tipo lipoatrófica, após ajuste para gordura corporal periférica e total. Isto provavelmente se deve à redução da gordura total e conseqüente redução da produção de leptina pelos adipócitos. Indivíduos com lipo-hipertrofia apresentaram os maiores níveis séricos de leptina, provavelmente devido a um estado de resistência à leptina ou excesso de produção de leptina pelo tecido adiposo. Contudo, ainda existem dados controversos na literatura quanto ao papel da leptina na $\operatorname{SLHIV}(83,84)$.

\section{Fisiopatologia da SLHIV}

A fisiopatologia da SLHIV ainda não foi totalmente elucidada. Contudo, existem algumas hipóteses que tentam explicar, através de diferentes mecanismos, a ação dos IPs e dos NRTIs na gênese da lipodistrofia do HIV.

Postula-se que os NRTIs causem lesão mitocondrial em diferentes linhagem celulares e por mecanismos diversos (85). Assim sendo, alguns NRTIs causariam alterações a partir da inibição da enzima DNA polimerase-g (enzima responsável pela replicação do DNA mitocondrial); enquanto outros NRTIs poderiam alterar a função mitocondrial através de diferentes mecanismos (85). Dentre os eventos adversos secundários à diminuição da função mitocondrial causada pelos NRTIs estão a acidose lática, neuropatia periférica, miopatias, esteatose hepática, hipogonadismo, diabetes, anemia e lipoatrofia (85).
Apesar de os mecanismos pelos quais os IPs determinam a SLHIV permanecerem desconhecidos, existem algumas hipóteses propostas (39). Alguns IPs prejudicam a diferenciação dos pré-adipócitos, levando à apoptose dos adipócitos subcutâneos, conforme demonstrado em biópsias musculares de indivíduos usuários de IP (86). Estudos demonstram que o tecido adiposo de pacientes que recebem IP apresenta uma redução na expressão de fatores de transcrição envolvidos na adipogênese, incluindo o sterol regulatory element-binding protein Ic (SREBPIc) e peroxisomeproliferator-activated receptor $\gamma(\operatorname{PPAR} \gamma)(87,88)$. Um recente estudo observou que alguns anti-retrovirais inibem a diferenciação e induzem resistência insulínica e apoptose em células adiposas in vitro e in vivo (35). In vitro, os IPs e os NRTIs aumentam a expressão e a secreção de citocinas pró-inflamatórias (TNF $\alpha$, IL-6 e L-1 $\beta$ ) envolvidas na modificação da função dos adipócitos e na redução da adiponectina. Alterações semelhantes são observadas no tecido adiposo e no soro de pacientes soropositivos tratados com IPs e NRTIs. Alterações na diferenciação dos adipócitos, resistência insulínica, apoptose e lipoatrofia provavelmente resultam na diminuição da secreção de adiponectina e no aumento dos ácidos graxos livres por resistência insulínica no tecido adiposo (89).

Inúmeros estudos estão em andamento para melhor elucidar a gênese da SLHIV.

\section{Diagnóstico, Tratamento e Seguimento da SLHIV}

Até o presente momento, a literatura mundial não dispõe de nenhum consenso que determine os critérios para o diagnóstico e o tratamento da SLHIV. Desta forma, as dúvidas de quando e como tratar a SLHIV ainda não foram esclarecidas. Atualmente, o tratamento baseia-se na experiência de pequenos estudos que utilizam os medicamentos disponíveis para o controle da dislipidemia e das alterações glicêmicas, na população soropositiva, conforme descrito anteriormente e resumido na tabela 2. Estudos bem desenhados, que acompanhem um grande número de pacientes por tempo prolongado são necessários para que sejam melhor conhecidos os riscos da interação destes medicamentos com a terapia anti-retroviral.

A tabela 3 apresenta sugestões para o seguimento das alterações metabólicas e do risco cardiovascular dos pacientes adultos soropositivos em terapia antiretroviral. Tais sugestões baseiam-se em um estudo recentemente publicado (31) e em nossa experiência no acompanhamento de pacientes com síndrome metabólica clássica e com a SLHIV. Embora auxiliem na prática 
Tabela 2. Possíveis intervenções na Síndrome Lipodistrófica do HIV (SLHIV).

\begin{tabular}{|c|c|}
\hline Intervenção & Comentários \\
\hline $\begin{array}{l}\text { Mudança na terapia ARV } \\
\text { Novo IP: Atazanavir }\end{array}$ & $\begin{array}{l}\text { - Risco de piora do controle da SIDA ( } \uparrow \text { da carga viral / } \downarrow \text { CD4) } \\
\text { - Droga com potência antiviral e segurança, que não altera o colesterol T e } \\
\text { triglicérides em pacientes virgens de tratamento ARV }(49,50) \\
\text { - Reduziu o colesterol T, LDL, triglicérides, gordura abdominal e gibosidade dorsal } \\
\text { de pacientes em uso de HAART (50-53) }\end{array}$ \\
\hline $\begin{array}{l}\text { Mudança do estilo de vida } \\
\text { Estatinas }\end{array}$ & $\begin{array}{l}\text { - Dieta e atividade física: melhora do perfil metabólico; melhora da lipodistrofia? } \\
\text { - Reduzem em cerca de } 25 \% \text { o colesterol total e LDL colesterol } \\
\text { - Evitar: sinvastatina, lovastatina e atorvastatina, as quais são metabolizadas pelo } \\
\text { citocromo P450. Preferir a fluvastatina ou a pravastatina (32) } \\
\text { - Controle rigoroso das enzimas hepáticas, função renal e CPK (10) }\end{array}$ \\
\hline & $\begin{array}{l}\text { - Reduzem em cerca de } 20-25 \% \text { os triglicérides } \\
\text { - Controle rigoroso das enzimas hepáticas, função renal e CPK (10) }\end{array}$ \\
\hline Metformina & $\begin{array}{l}\text { - } \downarrow \text { glicemia de jejum, } \downarrow \text { gordura visceral, } \downarrow \text { resistência insulínica (36-38); } \downarrow \text { PAI } 1 \text { e } \\
\text { tPA }(36,37) \\
\text { - Risco de acidose lática quando da associação com NRTls }\end{array}$ \\
\hline $\begin{array}{l}\text { Glitazonas Rosiglitazona } \\
(4 \mathrm{mg} / \text { dia) }\end{array}$ & $\begin{array}{l}\text { - } \uparrow \text { sensibilidade insulínica, } \uparrow \text { adiponectina, } \uparrow \text { gordura periférica e } \downarrow \text { ácidos graxos } \\
\text { livres (40) } \\
\text { - Composição corporal inalterada, } \uparrow \text { colesterol T e triglicérides (41) }\end{array}$ \\
\hline $\mathrm{GH}(4-6 \mathrm{mg} / \mathrm{d})$ & - Progressão para neoplasias malignas e alterações glicêmicas? \\
\hline
\end{tabular}

Tabela 3. Sugestões para o seguimento das alterações metabólicas e do risco cardiovascular de pacientes adultos soropositivos em terapia anti-retroviral.

Realizar avaliação:

- Antes da introdução da terapia anti-retroviral

- Anualmente durante a terapia anti-retroviral estável

- Após qualquer modificação no esquema anti-retroviral ${ }^{*}$

Avaliação:

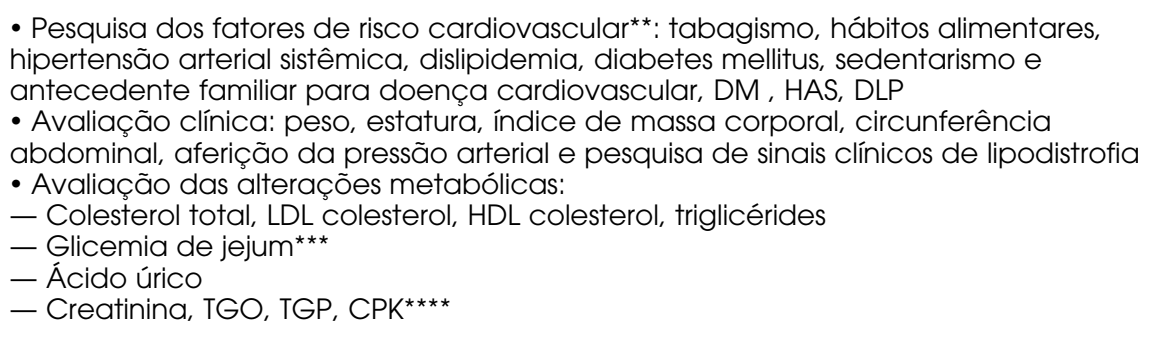

* Reavaliação lipídica de 1-2 meses após qualquer mudança no esquema anti-retroviral. Reavaliação glicêmica poucas semanas após qualquer mudança no esquema ARV.

** Identificar e corrigir fatores de risco modificáveis.

*** TOTG: deve ser realizado se a glicemia de jejum for $\geq 100 \mathrm{mg} / \mathrm{dl}$; para pacientes com fator de risco para diabetes do

tipo 2, para pacientes com outros fatores de risco cardiovascular ou para pacientes com lipoatrofia grave.

$* * * \star$ Importante realizar a monitorização destes exames para uma eventual necessidade de introduzir drogas como fibratos, estatinas ou hipoglicemiantes orais.

clínica, não substituem a avaliação individualizada do paciente por seu médico, nem tão pouco a eventual necessidade de avaliações mais abrangentes ou precoces.

\section{CONCLUSÕES E PERSPECTIVAS}

O papel da terapia ARV na evolução da SIDA é fato incontestável. Todavia, o emprego destas medicações propicia o desenvolvimento da síndrome metabólica associada ao HIV. A SLHIV eleva o risco de complicações metabólicas e cardiovasculares destes pacientes, alterando o paradigma da SIDA e colocando em contato com esta população outros profissionais da área médica como cardiologistas e endocrinologistas. $\mathrm{Na}$ medida em que aumenta a expectativa de vida destes indivíduos, a comunidade científica se depara com a instalação de outras co-morbidades que, a longo prazo, podem apresentar impacto na qualidade de vida e na mortalidade dos pacientes. Isto se torna ainda 
mais importante em indivíduos jovens e crianças que atualmente se beneficiam do emprego das terapias antivirais. A fisiopatologia das alterações metabólicas, bem como seus agentes causais, ainda não foram totalmente descobertos. Consensos específicos para o tratamento dos componentes da síndrome metabólica do HIV ainda não estão disponíveis. Assim sendo, somente uma adequada elucidação dos mecanismos fisiopatológicos da síndrome propiciará a escolha de medidas terapêuticas mais eficazes, reduzindo-se o risco cardiovascular secundário à SLHIV.

\section{AGRADECIMENTOS}

Angélica Marques Martins Valente recebeu uma bolsa de mestrado da CAPES durante o desenvolvimento deste trabalho.

\section{REFERÊNCIAS}

1. Sepkowitz KA. AIDS - The First 20 Years. $\mathbf{N}$ Engl J Med 2001;344(23): 1764-72

2. AIDS Epidemic Update: December 2002. World Health Organization Disponível em: http://www.who.int/hiv/pub/epidemiology/epi2002/en.

3. Epidemiologia da AIDS no Brasil. Ministério da Saúde do Brasil / Secretaria Executiva / Coordenação Nacional de DST e AIDS. Disponível em: http://www.henriqueser.org.br/aids_brasil.htm.

4. Gulick RM, Mellors JW, Havlir D, et al. Treatment with indinavir, zidovudine, and lamivudine in adults with human immunodeficiency virus infection and prior antiretroviral therapy. N Engl J Med 1997;337(1 1):734-9.

5. Palle Jr FJ, Delaney KM, Moorman AC, et al. Declining morbidity and mortality among patients with advanced human immunodeficiency virus infection. N Engl J Med 1998;338(13):853-60.

6. Behrens $G$, Dejam A Schmidt $H$, et al. Impaired glucose tolerance, beta cell function and lipid metabolism in HIV patients under treatment with protease inhibitors. AIDS 1999: 13:F63-70.

7. Carr A, Cooper DA. Adverse effects of antiretroviral therapy. Lancet 2000;356:1423-30.

8. Hadigan C, Meigs JB, Corcoran C, et al. Metabolic abnormalities and cardiovascular disease risk factors in adults UIT human immunodeficiency virus infection and lipodystrophy. Clin Infect Dis 2001;32:130-9.

9. Tanwani LK, Mokshagundam SPL. Lipodystrophy, insulin resistance, diabetes mellitus, dyslipidemia, and cardiovascular disease in human immunodeficiency virus infection. Southern Med J 2003;96(2):180-8.

10. Montessori V, Press $N$, Harris $M$, et al. Adverse effects of antiretroviral therapy for HIV infection. CMAJ 2004; 170(2):229-38.
11. Passalaris JD, Sepkowitz KA, Glesby MJ. Coronary artery disease and human immunodeficiency virus infection. Clin Infect Dis 2000:31:787-97.

12. Lumpkin M. FDA Public Health Advisory: Reports of diabetes and hyperglycemia in patients receiving protease inhibitors for the treatment of human immunodeficiency virus (HIV). Washington, DC: US Government Printing Office, 1997

13. "Crix-list". Disponível em: http://crix.pinkpage.com/. Acessado em 10 de novembro de 2003.

14. Miller KD, Jones E, Yanovski JA, et al. Visceral abdominal - Fat accumulation associated UIT use of indinavir. Lancet 1998;351:871-5.

15. Miller KK, Daly PA, Sentochnik D, et al. Pseudo-Cushing's syndrome in human immunodeficiency virus-infected patients. Clin Infect Dis 1998:27:68-72.

16. Lo JC, Mulligan K, Tai VW, et al. "Buffalo Hump" in men with HIV-1 infection. Lancet 1998;351:867-70.

17. Carr A. HIV protease inhibitor - Related lipodystrophy syndrome. Clin Infect Dis 2000;30(suppl. 2):5135-42.

18. Gervasoni C, Ridolfo AL, Trifirò $G$, et al. Redistribution of body fat in HIV-infected women undergoing combined antiretroviral therapy. AIDS 1999;13:465-71.

19. Hadigan C, Meigs JB, Wilson PWF, et al. Prediction of coronary heart disease risk in HIV-infected patients with fat redistribution. Clin Infect Dis 2003:36:909-16.

20. Balasubramanyan A, Sekhar RV, Jahoor F, et al. Pathophysiology of dyslipidemia and increased cardiovascular risk in HIV lipodystrophy: A model of "systemic steatosis". Curr Op Lipidol 2004; 15:59-67.

21. Grunfeld C, Kotler DP, Hamadeh R, et al. Hypertriglyceridemia in the acquired immunodeficiency syndrome. Am J Med 1989:86:27-31.

22. Périard $D$, Telenti $A$, Sudre $P$, et al. Atherogenic dyslipidemia in HIV-infected individuals treated with protease inhibitors. Circulation 1999; 100:700-5.

23. Carr A, Samaras K, Burton S, et al. A syndrome of peripheral lipodystrophy, hyperlipidaemia and insulin resistance in patients receiving HIV protease inhibitors. AIDS 1998; 12:F51-8

24. Tsiodras S, Mantzoros C, Hammer S, Samore M. Effects of protease inhibitors on hyperglycemia, hyperlipidemia, and lipodystrophy - A 5-year cohort study. Arch Inter Med 2000; 160:2050-6.

25. Heath K, Chan KJ, Singer J, et al. Incidence of morphological and lipid abnormalities: Gender and treatment differentials after initiation of first antiretroviral therapy. Intern J Epidemiol 2002;31:1016-20.

26. Madge S, Kinloch-de-Loes S, Mercey D, et al. Lipodystrophy in patients naive to HIV protease inhibitors (Correspondence). AIDS 1999;13(6):735-7.

27. Purnell J, Zambon A, Knopp R, et al. Effect of ritonavir on lipids and post-heparin lipase activities in normal subjects. AIDS 2000; 14:51-7.

28. Früs-Moller $N$, Weber $R$, Reiss $P$, et al. Cardiovascular disease risk factors in HIV patients - Association with antiretroviral therapy: Results from the DAD study. AIDS 2003: 17:1179-3. 
29. Schambelan M, Benson CA, Carr A, et al. Management of metabolic complications associated with antiretroviral therapy for HIV-1 infection: Recommendations of an international AIDS society - USA panel. J Acquir Immune Defic Syndr 2002;31:257-75.

30. National Cholesterol Education Program Executive summary of the third report of the National Cholesterol Education Program (NCEP) Expert Panel on Detection, Evaluation and Treatment of High Blood Cholesterol in adults (Adult treatment Panel III). JAMA 2001;285:248-97.

31. Grinspoon S, Carr A. Cardiovascular risk and body-fat abnormalities in HIV-infected adults. N Engl J Med 2005;352:48-62.

32. Fichtenbaum CJ, Gerber JG, Rosen Kranz SL, et al. Pharmacokinetic interactions between protease inhibitors and statins in HIV seronegative volunteers: ACTG Study A5047. AIDS 2002:16:569-77.

33. Lee ECC, Walmsley S, Fantus IG. New onset diabetes mellitus associated with preotease inhibitor therapy in an HIV-positive patient: Case report and review. CMAF 1999; 161(2):161-4

34. Noor MA, Lo JC, Mulligan K, et al. Metabolic effects of indinavir in healthy HIV-seronegative men. AIDS 2001;15:F11-8.

35. Dube MP. Disorders of glucose metabolism in patients infected with human immunodeficiency virus. Clin Infect Dis 2000;31:1467-75

36. Hadigan C, Corcoran C, Basgoz N, et al. Metformin in the treatment of HIV lipodystrophy syndrome - A randomized controlled trial. JAMA 2000;284(4):472-7.

37. Hadigan C, Meigs JB, Rabe J, et al. Increased PAI-1 and TPA antigen levels are reduced UIT metformin therapy in HIV-infected patients UIT fat redistribution and insulin resistance. J Clin Endocrinol Metab 2001;86(2):939-43.

38. Hadigan C, Rabe J, Grinspoon S. Sustained benefits of metformin therapy on markers of cardiovascular risk in human immunodeficiency virus-infected patients UIT fat redistribution and insulin resistance. J Clin Endocrinol Metab 2002;87(10):4611-5.

39. Garg A. Acquired and inherited lipodystrophies. N Engl J Med 2004;350(12):1120-34.

40. Hadigan C, Yawetz S, Thomas A, et al. Metabolic effects of rosiglitazone in HIV lipodystrophy. Ann Intern Med 2004; 140:786-94

41. Carr A, Workman C, Carey D, et al. Rosiglitazone does not improve antiretroviral-associated lipoatrophy. Lancet 2004;363:429-38.

42. Saint-Marc T, Partisani M, Poizot-Martin I, et al. A Syndrome of peripheral fat wasting (lipodystrophy) in patients receiving long-term nucleoside analogue therapy. AIDS 1999;13:1359-67.

43. Carr A, Miller J, Law M, Cooper DA. A syndrome of lipodystrophy, latic acidaemia and liver dysfunction associated with nucleoside analogue therapy: Contribution to protease inhibition -Related lipodystrophy syndrome. AIDS 2000; 14:F25-32.

44. Thiébaut R, Daucourt $V$, Mercié P. Lipodystrophy, metabolic disorders, and human immunodeficiency virus infection: Aquitaine cohort, France, 1999. Clin Infect Dis 2000;31:1482-7.
45. Carr A, Hudson J, Chuah J, et al. HIV protease inhibitors substitution in patients with lipodystrophy: A randomized, controlled, open-label, multicentre study. AIDS 2001;15:1811-22

46. Roubenoff $\mathrm{R}$, Schmitz $\mathrm{H}$, Bairos $\mathrm{L}$, et al. Reduction of abdominal obesity in lipodystrophy associated with human immunodeficiency virus infection by means of diet and exercise: Case report and proof of principle. Clin Infect Dis 2002;34:390-3.

47. Roubenoff $R$, Weiss $L$, McDermott $A$, et al. A pilot study of exercise training to reduce trunk fat in adults with HIVassociated fat redistribution. AIDS 1999;13:1373-5.

48. Schambelan M, Mulligan K, Greenfeld C, et al. Recombinant human growth hormone in patients with HIVassociated wasting. A randomized, placebo-controlled trial. Ann Intern Med 1996; 125:873-82.

49. Wolfort FG, Cetrulo CL Jr, Nevarre DR. Suction-assisted lipectomy for lipodystrophy syndromes attributed to HIV - Protease inhibitor use. Plast Reconstr Surg 1999: 104:1814-22.

50. Goldsmith DR, Perry CM. Atazanavir. Drugs 2003; 16:167993.

51. Sane I, Piliero $P$, Squires $K$, et al. Results of a phase 2 clinical trial at 48 weeks (Al 424-007) a dose-ranging safety, and efficacy comparative trial of atazanavir at three doses in combination with didanosine and stavudine in antiretroviral-naïve subjects. J Acquir Immune Defic Syndr 2003; 1:18-29.

52. Haas DW, Zala C, Schrader S, et al. Therapy with atazanavir plus saquinavir in the patients failing highly active antiretroviral therapy: A randomized comparative pilot trial. AIDS 2003:17:1339-49.

53. Haerter $G$, Manfras BJ, Mueller $M$, et al. Regression of lipodystrophy in HIV-infected patients under therapy with the new protease inhibitor atazanavir. AIDS 2004; 18(6):952-5.

54. Cotter B. Epidemiology of HIV cardiac disease. Progress Cardiov Dis 2003:45(4):319-26.

55. Barbaro G, Di Lorenzo G, Gresorio B, et al. Cardiac involvement in the acquired immunodeficiency syndrome: A multicentre clinical-pathological study. AIDS Res Hum Retrovir 1998:14:1071-7.

56. Yunis NA, Stone VE. Cardiac manifestations of HIV I AIDS: A review of disease spectrum and clinical manegement. J Acquir Immune Defic Syndr Hum Retrovirol 1998; 18:145-54

57. Rerkpattanapipat $\mathrm{P}$, Wongpraparut $\mathrm{N}$, Jacobs LE, et al. Cardiac manifestations of acquired immunodeficiency syndrome. Arch Intern Med 2000;160:602-8.

58. Henry K, Melroe H, Huebsch J, et al. Severe premature coronary artery disease with protease inhibitor (Letter). Lancet 1998:351:1328.

59. Rickerts V, Brodt H, Staszewski S, et al. Incidence of myocardial infarctions in HIV-infected patients between 1983 and 1998: The Frankfurt HIV-cohort study. Eur J Med Res 2000:5:329-33.

60. Barbaro G, Barbarini G, Pellicelli AM. HIV-associated coronary arteritis in a patient with fatal myocardial infarction. N Engl J Med 2001;344:1799-800. 
61. Flynn TE, Bricker LA. Myocardial infarction in HIV-infected men receiving protease inhibitors. Ann Intern Med 1999; 131:548.

62. Friedl AC, Attenhofer Jost $\mathrm{CH}$, Schalcher $\mathrm{C}$, et al. Acceleration of confirmed coronary artery disease among HIV-infected patients on potent antiretroviral therapy. AIDS 2000; 14:2790-2.

63. Joshi VV, Pawel B, Conner E, et al. Arteriopaty in children with AIDS. Pediatr Pathol 1987;7:261-75.

64. Paton P, Tabib A, Loire R, Tete R. Coronary artery lesions and human immunodeficiency virus infection. Res Virol 1993; 144:225-31.

65. Fuster V, Badimon L, Padimon JJ, Chesebro JJ. The progression of coronary artery disease and the acute coronary syndromes. N Engl J Med 1992;326:242-50.

66. Fuster V, Badimon L, Padimon JJ, Chesebro JJ. The progression of coronary artery disease and the acute coronary syndromes. N Engl J Med 1992;326:310-8.

67. Klein D, Hurley L, Sidney S. Do protease inhibitors increase the risk for coronary heart disease among HIVpositive patients? Additional follow-up. Proceedings of the $7^{\text {th }}$ Conference on Retroviruses and Opportunistic Infections. San Francisco, CA, January 30 - February 2 2000, p. 81 (abstr 33)

68. Bozzette SA, Ake CF, Tam HK, et al. Cardiovascular and cerebrovascular events in patients treated for human immunodeficiency virus infection. N Engl J Med 2003;348(8):702-10.

69. The Data Collection on Adverse Events of Anti-HIV Drugs (DAD) Study Group - Combination antiretroviral therapy and the risk of myocardial infarction. $\mathbf{N}$ Engl $\mathbf{J}$ Med 2003;349: 1993-2003

70. Alessi MC, Peiretti F, Morange $P$, et al. Production of plasminogen activator inhibitor 1 by human tissue: Possible link between visceral fat accumulation and vascular disease. Diabetes 1997:46:860-7.

71. Hotamisligil GS, Spiegelman BM. Tumor necrosis factor- $\alpha$ : A key component of the obesity-diabetes link. Diabetes 1994:43:1271-8

72. Hotamisligil GS, Murray DL, Chay LN. TNF- $\alpha$ inhibitors signaling from the insulin receptor. Proc Natl Acad Sci USA 1994:91:4854-8

73. Hanson K, Pratley RE. Interleukin-6 is associated UIT insulin resistance independently of obesity. Diabetes 2000;49(suppl. 1):A297(abstract).

74. Mantzoros CS. The role of leptin in human obesity and disease: A review of the current evidence. Ann Intern Med 1999:130:651-7.

75. Weyer C, Funahashi T, Tanaka S, et al. Hypoadiponectinemia in obesity and type 2 diabetes: Close association with insulin resistance and hyperinsulinemia. J Clin Endocrinol Metab 2001;86:1930-5.

76. Yamauchi T, Kamon J, Waki H, et al. The fat-derived hormone adiponectin reverses insulin resistance associated with both lipoatrophy and obesity. Nat Med 2001;7:941-

77. Haque WA, Shimonura I, Matsuzawa Y, et al. Serum adiponectin and leptin levels in patients with lipodystrophies. J Clin Endocrinol Metab 2002;87:2395-8.
78. Addy CL, Gavrila A, Tsiodras S, et al. Hypoadiponectinemia is associated with insulin resistance, hypertriglyceridemia, and fat redistribuition in human immunodeficiency virus-infected patients treated with highly active antiretroviral therapy. J Clin Endocrinol Metab 2003;88(2):627-36

79. Nagy GS, Tsiodras S, Martin LD, et al. Human immunodeficiency virus type 1-related lipoatrophy and lipohypertrophy are associated with serum concentrations of leptin. Clin Infect Dis 2003:36:795-802.

80. Lonnqvist F, Arner P, Nordfors $L$, et al. Over expression of the obese (ob) gene in adipose tissue of human obese subjects. Nat Med 1995; 1:950-3.

81. Considine RV, Sinha MK, Heiman ML, et al. Serum immunoreactive-leptin concentrations in normal weight and obese human. N Engl J Med 1996;334:292-5.

82. Oral EA, Sinha V, Ruez E, et al. Leptin-replacement therapy for lipodystrophy. N Engl J Med 2002;346:570-8.

83. Estrada V, Serrano-Rios M, Larrada MTM, et al. Leptin and adipose tissue maldistribuition in HIV-infected male patients with predominant fat loss treated with antiretroviral therapy. J Acquir Immune Defic Syndr 2002;29:3240.

84. Christeff N, Melchior JC, de Truche P, et al. Lipodystrophy defined by a clinical score in HIV-infected men on highly active antiretroviral therapy: Correlation between dyslipidemia and steroid hormone alterations. AIDS 1999; 13:2251-60.

85. Cossariza A, Moyle G. Antiretroviral nucleoside and nucleotide analogues and mitochondria. AIDS 2004; 18:137-51.

86. Domingo P, Malia-Guiu X, Pujol RM, et al. Subcutaneous adipocyte apoptosis in HIV-1 protease inhibitor-associated lipodystrophy. AIDS 1999; 13:2261-7.

87. Bastard JP, Caron M, Vidal $\mathrm{H}$, et al. Association between altered expression of adipogenese factor SREBPIc in lipoatrophic adipose tissue from HIV-1-infected patients and abnormal adipocyte differentiation and insulin resistance. Lancet 2002;359:1026-31.

88. Shimomura I, Hammer RE, Richardson JH, et al. Insulin resistance and diabetes mellitus in transgenic mice expressing nuclear SREBPIc in adipose tissue: Model for congenital generalized lipodystrophy. Genes Dev 1998:12:3182-94.

89. Lagathu C, Kim M, Maachi M, et al. HIV antiretroviral treatment alters adipokine expression and insulin sensitivity of adipose tissue in vitro and in vivo. Biochimie 2005 (online abstract)

\section{Endereço para correspondência:}

Angélica Marques Martins Valente

Rua Pedro de Toledo 544, apto. 806

04039-001 São Paulo, SP

Fax: (1 1) 5908-1523

E-mail: angelicammvalente@uol.com.br 